\title{
Application of an electronic tongue as a single-run tool for olive oils' physicochemical and sensory simultaneous assessment
}

\author{
Nuno Rodrigues ${ }^{\mathrm{a}}$, Ítala M.G. Marx ${ }^{\mathrm{b}}$, Susana Casal ${ }^{\mathrm{c}}$, Luís G. Dias ${ }^{\mathrm{a}}$, Ana C.A. Veloso ${ }^{\mathrm{d}, \mathrm{e}}$, \\ José A. Pereira ${ }^{\mathrm{a}}$, António M. Peres ${ }^{\mathrm{a}, \mathrm{b}, *}$ \\ ${ }^{a}$ Centro de Investigação de Montanha (CIMO), ESA, Instituto Politécnico de Bragança, Campus Santa Apolónia, 5300-253 Bragança, Portugal \\ ${ }^{\mathrm{b}}$ Laboratory of Separation and Reaction Engineering - Laboratory of Catalysis and Materials (LSRE-LCM), ESA, Instituto Politécnico de Bragança, Campus Santa Apolónia, \\ 5300-253 Bragança, Portugal \\ ${ }^{\mathrm{c}}$ LAQV/REQUIMTE, Laboratory of Bromatology and Hydrology, Faculty of Pharmacy, University of Porto, Rua de Jorge Viterbo Ferreira, 228, 4050-313 Porto, Portugal \\ ${ }^{\mathrm{d}}$ Instituto Politécnico de Coimbra, ISEC, DEQB, Rua Pedro Nunes, Quinta da Nora, 3030-199 Coimbra, Portugal \\ ${ }^{\mathrm{e}}$ CEB - Centre of Biological Engineering, University of Minho, Campus de Gualtar, 4710-057 Braga, Portugal
}

\section{A R T I C L E I N F O}

\section{Keywords:}

Olive oil

Physicochemical-sensory analysis

Electronic tongue

Chemometrics

Predictive performance

\begin{abstract}
A B S T R A C T
Olive oil is highly appreciated due to its nutritional and organoleptic characteristics. However, a huge compositional variation is observed between olive oils, requiring the use of diverse analytical techniques for its classification including titration, spectrophotometry and chromatography, as well as sensory analysis. Chemical analysis is usually time-consuming, expensive and require skilled technicians, while the sensorial ones are dependent upon individual subjective evaluations, even if performed by trained panellists. This work evaluated and demonstrated the feasibility of using a potentiometric electronic tongue, comprising non-specific lipid polymeric and cross-sensitive sensor membranes, coupled with chemometric tools based on different sub-sets of sensors (from 11 to 14 sensors), to predict key quality parameters of olive oils based on single-run assays. The multivariate linear models established for 23 centenarian olive trees from different cultivars allowed predicting peroxide value, oxidative stability, total phenols and tocopherols contents, CIELAB scale parameters $\left(\mathrm{L}^{*}, \mathrm{a}^{*}\right.$ and $\mathrm{b}^{*}$ values), as well as 11 gustatory-retronasal positive attributes (green, sweet, bitter, pungent, tomato and tomato leaves, apple, banana, cabbage, fresh herbs and dry fruits) with satisfactory accuracy $\left(0.90 \pm 0.07 \leq \mathrm{R}^{2}\right.$ $\leq 0.98 \pm 0.02$ for the repeated K-fold-CV procedure, which ensured that $25 \%$ of the data was used for internalvalidation purposes). The electronic tongue device had an accuracy statistically similar to that achieved with standard analytical techniques, pointing out the versatility of the device for the fast and simultaneous chemical and sensory analysis of olive oil.
\end{abstract}

\section{Introduction}

Olive oil plays a key role in the Mediterranean diet, regarded as one of the healthiest worldwide due to its proved association with a lower risk for several chronic diseases, particularly cardiovascular ones [1]. To assure olive oil physicochemical and sensory quality as well as to minimize the risk of fraudulent practices like mislabelling or to enable health claims, it is legally required to evaluate different chemical parameters (e.g., free acidity, peroxide values, extinction coefficients, fatty acid composition, phenolic compounds, etc.) and sensory attributes (e.g., fruitiness intensity sensation and absence/presence of organoleptic defects) [2-6]. However, this characterization requires the use of various official standard analytical techniques, including titrations, spectrophotometry and chromatography, as well as the availability of official sensory panels. Still, these methodologies are, in most cases, time-consuming and/or expensive, requiring sample pretreatments, expensive equipment and skilled technicians [7], which turns them unaffordable for most traditional worldwide olive oil producers.

Therefore, several research groups have been trying to overcome some of these economic and technical limitations, by developing fast, accurate, cost-effective and user-friendly sensor-based analytical devices, namely the so-called electronic tongues (E-tongues) [8] or electronic noses (E-noses) [9]. In fact, the use of electrochemical devices (e.g., potentiometric and voltammetric ones) has been widely reported for food analysis and, more specifically for olive oils evaluation. For

\footnotetext{
*Corresponding author at: Centro de Investigação de Montanha (CIMO), ESA, Instituto Politécnico de Bragança, Campus Santa Apolónia, 5300-253 Bragança, Portugal.

E-mail address: peres@ipb.pt (A.M. Peres).
} 
Table 1

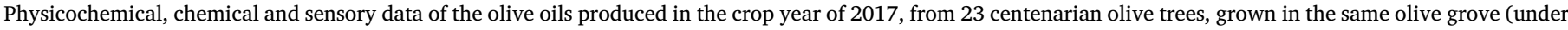

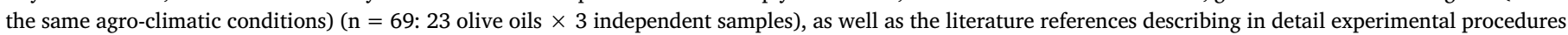
used.

\begin{tabular}{|c|c|c|c|c|}
\hline \multicolumn{2}{|l|}{ Chemical compound } & Experimental data range & Analytical method & Literature references \\
\hline \multicolumn{2}{|l|}{ Peroxide value ( $\mathrm{mEq} \mathrm{O}_{2} / \mathrm{kg}$ olive oil) } & {$[1.66,6.64]$} & Titration & {$[2]$} \\
\hline \multicolumn{2}{|l|}{ Oxidative stability (h) } & {$[14.5,35.0]$} & Rancimat & {$[20]$} \\
\hline \multicolumn{2}{|c|}{ Total phenols (mg gallic acid equivalents/kg olive oil) } & {$[217.6,541.2]$} & $\begin{array}{l}\text { HPLC with diode array detector and a C18 reversed- } \\
\text { phase column }\end{array}$ & {$[33,34]$} \\
\hline \multicolumn{2}{|l|}{ Total tocopherols (mg/kg olive oil) } & {$[162.1,536.0]$} & $\begin{array}{l}\text { HPLC with a fluorescence detector and a } \\
\text { SupelcosilTM LC-SI column }\end{array}$ & {$[35,36]$} \\
\hline \multirow[t]{3}{*}{ CIELAB colour scale ${ }^{a}$} & $L^{*}$ & {$[46.1,75.0]$} & Colorimeter & [37] \\
\hline & $a^{*}$ & {$[-18.0,-10.0]$} & & \\
\hline & $b^{*}$ & {$[38.7,81.3]$} & & \\
\hline \multirow{11}{*}{$\begin{array}{l}\text { Retronasal-gustatory positive sensory } \\
\quad \text { intensities }^{\text {b }}\end{array}$} & Green sensation & {$[5.3,8.6]$} & Sensory panel & {$[2-5]$} \\
\hline & Sweet sensation & {$[0.8,8.2]$} & & \\
\hline & Bitter sensation & {$[2.0,7.3]$} & & \\
\hline & Pungent sensation & {$[2.4,8.3]$} & & \\
\hline & Tomato sensation & {$[4.0,7.2]$} & & \\
\hline & Apple sensation & {$[0.0,5.9]$} & & \\
\hline & Banana sensation & {$[0.0,6.3]$} & & \\
\hline & Dry fruits sensation & {$[1.0,3.8]$} & & \\
\hline & Fresh herbs sensation & {$[2.3,6.2]$} & & \\
\hline & $\begin{array}{l}\text { Tomato leaves } \\
\text { sensation }\end{array}$ & {$[2.2,6.6]$} & & \\
\hline & Cabbage sensation & {$[0.0,7.4]$} & & \\
\hline
\end{tabular}

${ }^{\text {a }}$ CIELAB colour scale: $L^{*}$ value corresponds to lightness (0-black to 100-perfect reflector diffuser); $a^{*}$ negative value (green) and positive value (red); $b *$ negative values (blue) to positive values (yellow).

b Sensory analysis was performed by a trained sensory panel comprising 8 trained panellists of the School of Agriculture of the Polytechnic Institute of Bragança (Portugal), following the IOC regulations [4,5] (intensity scale: from 0 (absence of attribute) to 10 (maximum attribute intensity)).

example, as recently reviewed by Majchrzak et al. [9], E-noses have been widely used for classification and quality control of edible oils, being successfully applied for assessing their geographical origin, detecting adulteration practices as well as deterioration caused by external factors. Regarding E-tongues, several applications have been successfully reported, namely for the classification of olive oils according to the quality grade, geographical origin or olive cultivar, olive oil adulteration with other vegetable oils, refined oils or defective olive oils, detection of the addition of legally restricted or forbidden additives to olive oil, olive oil sensory evaluation (both positive and negative organoleptic sensations), olive oil shelf-life and quality trend during storage, olive oil quality parameters, polyphenolic, carotenoids and tocopherols contents, among other applications [10-32].

However, despite the proven successful use of electrochemical devices for olive oils analysis, industrial partners are still sceptic in applying these sensor-based methodologies as practical routine analytical tools. A tentative explanation would be that most of these works reported qualitative approaches, which although quite relevant, do not allow a straightforward assessment, in a quantitative manner, of the olive oil chemical composition neither of its physicochemical or sensory quality, therefore not fully disabling the need for chemical analysis. Indeed, Semenov et al. [28] pointed out recently the need to further explore the capacity of these electrochemical devices, namely the potentiometric ones, for the quantitative evaluation of particular chemical olive oil quality parameters. Besides, the possibility of using such electrochemical devices for establishing both physicochemical and sensory profiles based on a single-assay, which would be of utmost practical and economical relevance for olive oil producers and consumers, has not yet been evaluated.

Thus, this study aims to further evaluate the versatility of a potentiometric E-tongue, comprising two sensors arrays, each with 20 lipid polymeric membranes, for quantifying, in a single-run, several quality parameters associated with sensory, nutritional and health attributes of olive oils. For this, the potentiometric signal profiles of olive oil hydroethanolic extracts, which are rich in polar compounds, were processed using chemometric quantitative tools (e.g., multiple linear regression models) coupled with variable selection algorithms (e.g., meta-heuristic simulated annealing algorithm) aiming establishing predictive multivariate models.

\section{Materials and methods}

\subsection{Olive oil samples and quality grade}

As previously described in detail by Rodrigues et al. [33], the olive oils were extracted in a pilot plant with an Abencor analyzer (Comercial Abengoa S.A., Seville, Spain), from olives collected, during the 2017 crop season, from one farm with centenarian trees ( $>250$ years). The

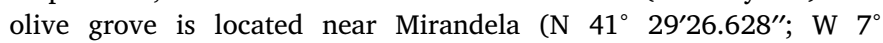
$\left.15^{\prime} 31.219^{\prime \prime}\right)$, in the northeast of Portugal. In total, 23 trees were selected among 140 trees, belonging to different olive cultivars, namely cvs. Lentisca ( 3 trees), Madural ( 3 trees), Rebolã ( 3 trees), Redondal (2 trees), Verdeal (2 trees), Verdeal Transmontana (7 trees) and nonidentified cultivars ( 3 trees). The olive oils were stored in $125 \mathrm{~mL}$ dark bottles in the dark and at room temperature. All the assays were carried out in triplicate within two months after extraction.

\subsection{Olive oils physicochemical, chemical and sensory data}

All olive oils were subjected to a complete set of physicochemical, chemical and sensory analysis, including peroxide values quantification, oxidative stability assays, total phenols and tocopherols levels assessment, olive oil colour scale parameters evaluation as well as perception of the intensity of positive organoleptic attributes. However, since the aim of this work was to evaluate the capability of an E-tongue to quantitatively assess olive oils characteristics, determined using different techniques (e.g., titration, UV/Vis spectrophotometry, colorimeter, liquid chromatography and sensory panel evaluation), only the parameters that showed wide dynamic ranges were considered. So, in summary, the parameters/attributes evaluated in this study are given in Table 1 together with the experimental data range and the references [2-5,20,33-37] describing, in detail, the analytical procedures used. Furthermore, according to the EU regulations and IOC recommendations [2-5] all olive oils were classified as extra virgin olive oils (EVOO) 


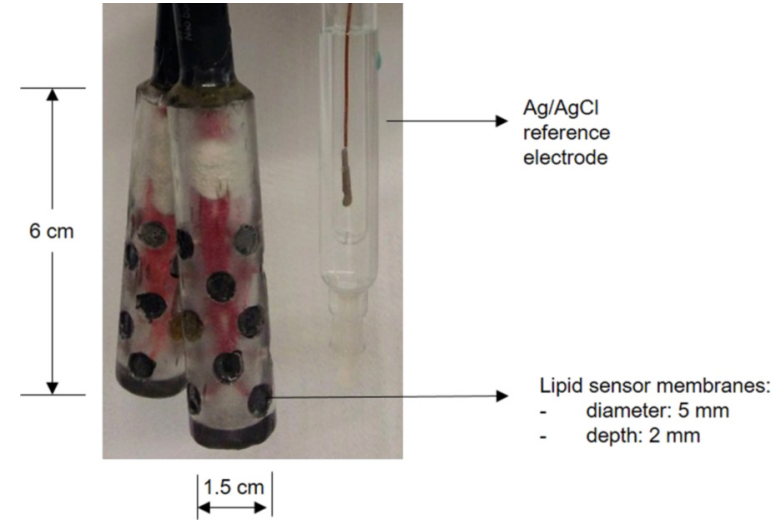

Fig. 1. E-tongue sensors arrays and $\mathrm{Ag} / \mathrm{AgCl}$ reference electrode.

since (data not shown): free acidity $\leq 0.8 \%$ oleic acid, peroxide values $\leq 20 \mathrm{mEq} \mathrm{O}{ }_{2} / \mathrm{kg}, K_{232} \leq 2.50, K_{270} \leq 0.22,|\Delta K| \leq 0.01$; ripe fruit median intensity greater than 0 and median intensity of defects equal to 0 .

\subsection{E-tongue}

\subsubsection{E-tongue device and set-up}

A new lab-made potentiometric E-tongue multisensor device, comprising two cylindrical arrays was built (Fig. 1). Each array contained 20 lipid polymeric cross-sensitive sensor membranes (40 sensors in total), which composition (lipid additive, 3\%; plasticizer, 32\%; and, polyvinyl chloride, 65\%) was the same as that used in the screenprinted arrays previously reported by the research team (e.g. [22-25]). However, aiming to enhance signal stability and detection performance, the membranes applied in the new device had higher diameter and thickness compared to the previous ones [22-25], allowing increasing the contact surface area. Similarly, the sensor membrane was connected to a multiplexer Agilent Data Acquisition Switch Unit (model 34970A) controlled by the Agilent BenchLink Data Logger software installed on a PC. Each potentiometric assay took $5 \mathrm{~min}$ and allowed recording the potentiometric signal profiles of the 40 sensor membranes generated through the establishment of electrostatic or hydrophobic interactions [38]. A reference $\mathrm{Ag} / \mathrm{AgCl}$ double-junction glass electrode (Crison, 5241) was used. Since the device was new, the signal repeatability during the usual 1-day period analysis was checked using basic taste aqueous standard solutions (acid: [citric acid] $=0.10 \mathrm{~g} / \mathrm{L}$; bitter: [caffeine] $=0.13 \mathrm{~g} / \mathrm{L}$; salty: [sodium chloride] $=0.175 \mathrm{~g} / \mathrm{L}$; sweet: [sucrose $]=1.75 \mathrm{~g} / \mathrm{L}$ ) as well as two olive oil hydroethanolic extracts (water-ethanol solutions, 80:20, v/v). After each analysis day, the two sensor arrays were stored by immersion in a $\mathrm{HCl}$ solution $(0.01 \mathrm{M})$. The same sensor coding used in previous works was adopted: each sensor was identified with a letter $S$ (for sensor) followed by the number of the array ( 1 or 2$)$ and the number of the membrane (1-20, corresponding to different combinations of plasticizers and additives).

\subsubsection{E-tongue analysis: olive oil sample preparation and potentiometric} assays

As previously described [21-25], the E-tongue analysis requires olive oil extraction $(10 \mathrm{~g})$ using $100 \mathrm{~mL}$ of water-ethanol solution $(80: 20, \mathrm{v} / \mathrm{v})$ to overcome the difficulty performing electrochemical assays in non-conductive and highly viscous liquids [19,39]. Besides, olive oils' hydroethanolic extracts are rich in polar compounds, including phenolic compounds, esters, alcohols and aldehydes, which are responsible for several sensory positive attributes like bitter, pungent, sweet, green, apple, banana, dry fruits (e.g., almonds) sensations $[22,23]$. Also, the use of hydroethanolic solutions promotes the tocopherols extraction since the addition of ethanol in the water results in a large increase in their solubility, mostly due to nonpolar attractive interactions between ethanol and the tocopherols [40]. Moreover, chlorophylls and carotenoids, the two type of pigments responsible for the greenness and yellowness oil colours [41], are soluble in alcohols or in water, respectively, and so, they can be extracted using the hydroethanolic solutions. The mixture (olive oil plus hydroethanolic solution) is agitated during 1-2 min using a vortex stirrer (LBX V05 series, lbx instruments) at $500 \mathrm{rpm}$. Then, it is left at ambient temperature for $60 \mathrm{~min}$, after which, $60 \mathrm{~mL}$ of the supernatant solution was carefully withdrawn and immediately analysed with the E-tongue, during a 5min period, which allowed reaching a pseudo-equilibrium between the non-specific lipid polymeric membranes comprised in the E-tongue and the chemical compound of the extract. Electrochemical assays were performed in duplicate for each sample, with a third assay carried out if the potentiometric signal of any of the 40 sensors showed a coefficient of variation greater than $20 \%$ (value set according to the IOC regulations for sensory analysis).

\subsection{Statistical analysis}

Multiple linear regression (MLR) models were used to estimate and/ or predict the experimental contents of the different physicochemical, chemical and sensory properties of olive oils (evaluated using titration, UV/Vis spectrophotometry, colorimeter, liquid chromatography and/or sensory evaluation by trained panellists), based on the potentiometric E-tongue signal profiles recorded during the analysis of the hydroethanolic extracts. The best number of sensors, with non-collinear potentiometric signals, used for quantitative purpose, was established by applying the simulated annealing (SA) meta-heuristic algorithm, which selection performance was previously demonstrated by the research team for MLRM-SA-E-tongue models [18,20,22,42]. The quality of the multivariate models was assessed through the coefficient of determination $\left(\mathrm{R}^{2}\right)$ and the root-mean-square error (RMSE), calculated two cross-validation (CV) variants: the leave-one-out CV (LOO-CV) and the repeated K-fold-CV procedures. This latter variant is less prone to overfitting issues since it allows to leave $1 / \mathrm{K} \times 100 \%$ of the initial data for internal-validation purposes (in this study, $\mathrm{K}$ was set equal to 4 , enabling that at each run, $25 \%$ of the initial data were used for validation purposes, i.e., olive oils produced from 5 to 6 independent centenarian olive trees of a total of 23 trees), being the data split process randomly repeated, which was set equal to 10 in this work $[18,20,22]$. Finally, the possibility of using the selected E-tongue-MLRSA models (for both LOO-CV and repeated K-fold-CV procedures) as complementary tools for the analytical conventional assessment of the physicochemical, chemical and sensory data was further checked, as suggested by Roig and Thomas $[43,44]$. Accordingly, the proposed MRLM-SA-E-tongue approach could be foreseen as a satisfactory tool if the $95 \%$ interval of confidence of the linear regression (LR) parameters (intercept and slope regression values of predicted versus experimental value) contained the theoretic ideal values of "zero" and "one" [43,44]. All statistical analyses were performed using the Subselect $[45,46]$ and MASS [47] packages of the open source statistical program R (version 2.15.1), at a $5 \%$ significance level.

\section{Results and discussion}

\subsection{E-tongue signal stability over time}

Potentiometric sensors may present signal drifts during the assay time-period, which could pose some limitations to the straightforward application of the methodology. This behaviour can be minimized or even overcome by daily calibrations or by applying signal normalization statistical procedures. Based on the previous experience of the research team, when similar potentiometric E-tongues were used, comprising the same lipid polymeric sensor membranes, the signals recorded during one-day of analysis were very stable, with negligible 


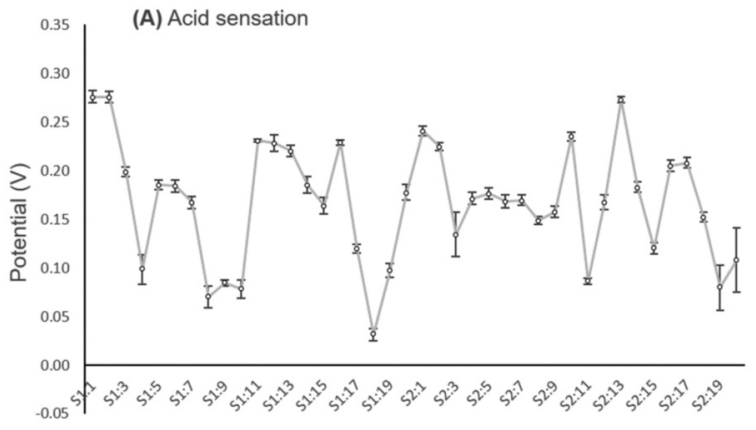

E-tongue sensors number

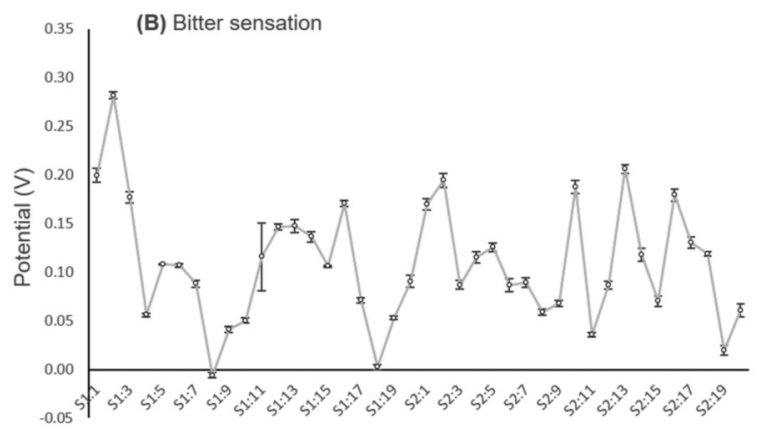

E-tongue sensors number

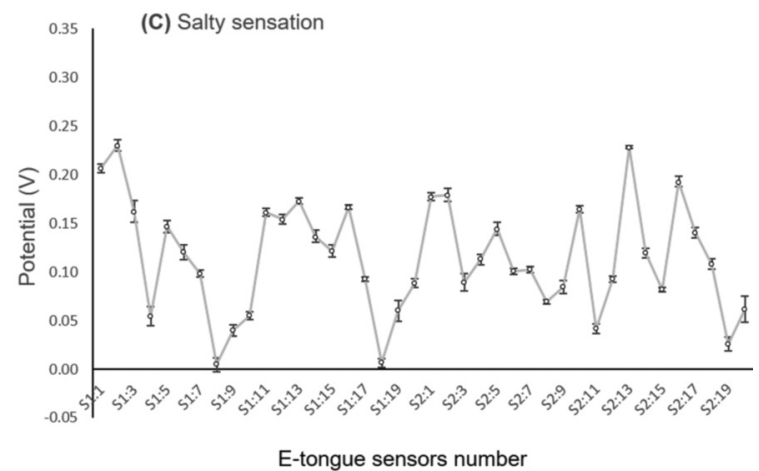

(D) Sweet sensation

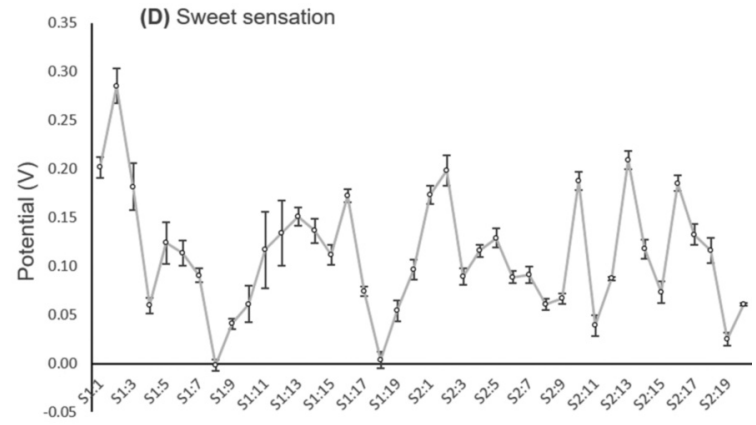

E-tongue sensors number

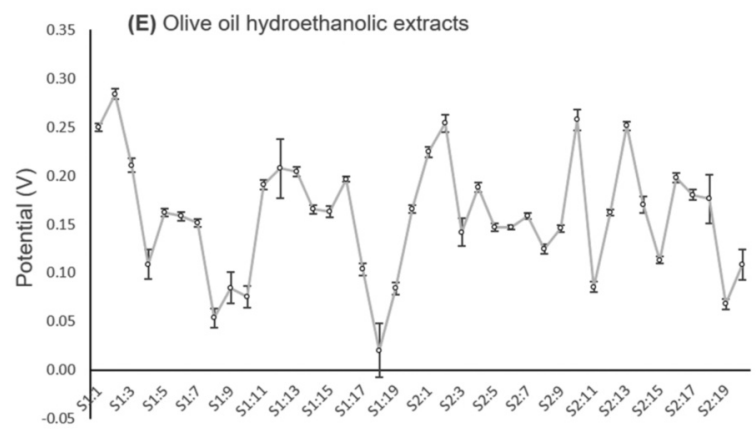

E-tongue sensors number

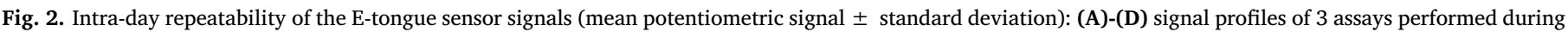

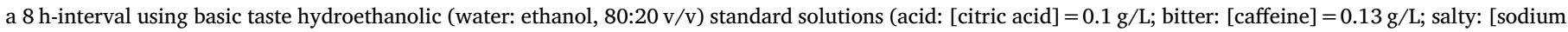

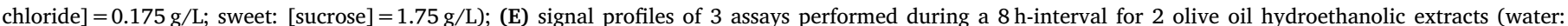
ethanol, $80: 20 \mathrm{v} / \mathrm{v})$.

signal drifts (with variation coefficients lower than 5\%) [22-25,48]. Nevertheless, since the new potentiometric E-tongue had a different geometric shape (cylindrical versus parallelepipedic form) and the membranes were larger and thicker, the repeatability of the potentiometric signals was checked using basic taste standard solutions (acid, bitter, salty and sweet aqueous solutions) as well as with hydroethanolic duplicate extracts of a selected olive oil randomly selected sample. All the above mentioned standard solutions and oil extracts were analysed three times within an $8 \mathrm{~h}$ time-period. The average signal recorded by each of the $40 \mathrm{E}$-tongue sensors regarding the 3 triplicate assays carried out in the same day together with the respective standard deviations (error bars) are shown in Fig. 2. As can be inferred, the potentiometric signals gathered by the 40 sensors that comprised the new E-tongue showed a satisfactory signal repeatability (in general, the coefficients of variation, CV\%, ranged between $1 \%$ and $5 \%$ ) and no significant signal drifts were observed. Also, for each kind of standard solution, which mimics the acid, bitter, salty or sweet basic taste, slightly different signal profiles (potential trends and the potential values as can be seen from Figs. 2A to 2D) were recorded by the sensor arrays, confirming that the device could be used as a taste sensor tool. Moreover, the average signals gathered by each of the 40 E-tongue sensors during the olive oil extract analysis (Fig. 2E) showed a satisfactory repeatability (in general, $\mathrm{CV} \% \leq 5 \%$ ). Furthermore, a similar signal trend was obtained, compared to the signal profiles recorded for the basic taste standard solutions, which could be attributed to the response of the sensor membranes towards different polar compounds present in the hydroethanolic extract that are responsible for typical olive oil sensations like bitter, sweet and green.

3.2. E-tongue quantitative performance: predictive capability for assessing physicochemical, chemical and sensory characteristics of olive oils produced from centenarian trees of different cultivars

The performance of the proposed potentiometric E-tongue, comprising cross-sensitive sensors, for simultaneously determining olive oils' peroxide value, oxidative stability, total phenols and total 
Table 2

Predictive capability of the E-tongue-MLR-SA models established to quantify physicochemical, chemical and sensory data of olive oils produced in the crop year of 2017, from 23 centenarian olive trees, grown in the same olive grove (under the same agro-climatic conditions) $(n=69: 23$ olive oils $\times 3$ independent samples).

\begin{tabular}{|c|c|c|c|c|c|}
\hline \multirow{3}{*}{$\begin{array}{l}\text { Chemical } \\
\text { compound/ } \\
\text { attribute }\end{array}$} & \multicolumn{5}{|c|}{ E-tongue-MLR-SA models ${ }^{\mathrm{a}}$} \\
\hline & \multirow[t]{2}{*}{$\begin{array}{l}\mathbf{N}^{\circ} \text { of } \\
\text { sensors }^{b}\end{array}$} & \multicolumn{2}{|c|}{$\begin{array}{l}\text { Determination } \\
\text { coefficient }\left(R^{2}\right)\end{array}$} & \multicolumn{2}{|c|}{$\begin{array}{l}\text { Root-mean-square } \\
\text { errors (RMSE) }\end{array}$} \\
\hline & & $\begin{array}{l}\text { LOO- } \\
\mathrm{CV}^{\mathrm{c}}\end{array}$ & $\begin{array}{l}\text { Repeated } K \text { - } \\
\text { fold-CV }{ }^{\mathrm{d}}\end{array}$ & $\begin{array}{l}\mathrm{LOO}- \\
\mathrm{CV}^{\mathrm{c}}\end{array}$ & $\begin{array}{l}\text { Repeated } K \text { - } \\
\text { fold-CV }\end{array}$ \\
\hline \multicolumn{6}{|c|}{ Physicochemical parameters } \\
\hline $\begin{array}{l}\text { Peroxide value (mEq } \\
\mathrm{O}_{2} / \mathrm{kg} \text { olive oil) }\end{array}$ & $12^{g}$ & 0.97 & $0.95 \pm 0.05$ & 0.21 & $0.27 \pm 0.10$ \\
\hline $\begin{array}{l}\text { Oxidative stability } \\
\text { (h) }\end{array}$ & $13^{\mathrm{h}}$ & 0.98 & $0.95 \pm 0.05$ & 1.3 & $1.7 \pm 0.8$ \\
\hline $\begin{array}{l}\text { Total phenols (mg } \\
\text { CAE/kg olive } \\
\text { oil) }\end{array}$ & $12^{\mathrm{i}}$ & 0.98 & $0.92 \pm 0.07$ & 16 & $22 \pm 8$ \\
\hline $\begin{array}{l}\text { Total tocopherols } \\
\text { (mg/kg olive oil) }\end{array}$ & $13^{j}$ & 0.98 & $0.93 \pm 0.07$ & 20 & $28 \pm 16$ \\
\hline \multicolumn{6}{|l|}{ Colour scale } \\
\hline CIELAB & $13^{\mathrm{k}}$ & 0.99 & $0.97 \pm 0.03$ & 1.2 & $1.6 \pm 0.9$ \\
\hline scale $^{\mathrm{e}}$ & $13^{1}$ & 0.97 & $0.93 \pm 0.06$ & 0.53 & $0.70 \pm 0.28$ \\
\hline$b^{*}$ & $13^{\mathrm{m}}$ & 0.98 & $0.96 \pm 0.05$ & 1.9 & $2.4 \pm 1.1$ \\
\hline \multicolumn{6}{|c|}{ Sensory gustatory-retronasal sensations ${ }^{f}$} \\
\hline $\begin{array}{c}\text { Green intensity } \\
\text { sensation }\end{array}$ & $13^{\mathrm{n}}$ & 0.99 & $0.98 \pm 0.02$ & 0.14 & $0.15 \pm 0.06$ \\
\hline $\begin{array}{c}\text { Sweet intensity } \\
\text { sensation }\end{array}$ & $14^{\circ}$ & 0.97 & $0.91 \pm 0.07$ & 0.39 & $0.50 \pm 0.26$ \\
\hline $\begin{array}{l}\text { Bitter intensity } \\
\text { sensation }\end{array}$ & $13^{\mathrm{p}}$ & 0.98 & $0.95 \pm 0.04$ & 0.25 & $0.44 \pm 0.31$ \\
\hline $\begin{array}{l}\text { Pungent intensity } \\
\text { sensation }\end{array}$ & $13^{\mathrm{q}}$ & 0.99 & $0.96 \pm 0.04$ & 0.23 & $0.40 \pm 0.21$ \\
\hline $\begin{array}{c}\text { Tomato intensity } \\
\text { sensation }\end{array}$ & $14^{\mathrm{r}}$ & 0.98 & $0.95 \pm 0.05$ & 0.16 & $0.21 \pm 0.12$ \\
\hline $\begin{array}{c}\text { Apple intensity } \\
\text { sensation }\end{array}$ & $12^{\mathrm{s}}$ & 0.99 & $0.94 \pm 0.06$ & 0.19 & $0.24 \pm 0.07$ \\
\hline $\begin{array}{c}\text { Banana intensity } \\
\text { sensation }\end{array}$ & $13^{t}$ & 0.99 & $0.96 \pm 0.04$ & 0.30 & $0.38 \pm 0.17$ \\
\hline $\begin{array}{l}\text { Dry fruits intensity } \\
\text { sensation }\end{array}$ & $11^{\mathrm{u}}$ & 0.97 & $0.94 \pm 0.05$ & 0.22 & $0.26 \pm 0.11$ \\
\hline $\begin{array}{l}\text { Fresh herbs intensity } \\
\quad \text { sensation }\end{array}$ & $11^{\mathrm{v}}$ & 0.94 & $0.90 \pm 0.07$ & 0.34 & $0.40 \pm 0.20$ \\
\hline $\begin{array}{c}\text { Tomato leaves } \\
\text { intensity } \\
\text { sensation }\end{array}$ & $13^{\mathrm{w}}$ & 0.99 & $0.98 \pm 0.04$ & 0.12 & $0.21 \pm 0.13$ \\
\hline $\begin{array}{c}\text { Cabbage intensity } \\
\text { sensation }\end{array}$ & $12^{\mathrm{x}}$ & 0.97 & $0.91 \pm 0.08$ & 0.54 & $0.64 \pm 0.32$ \\
\hline
\end{tabular}

a Multivariate linear regression (MLR) model based on sub-sets of potentiometric sensors, established using the simulated annealing (SA) algorithm, selected among the 40 possible signal profiles obtained with the electronic tongue (E-tongue) during the analysis of the olive oil hydroethanolic extracts.

b Number of signals included in the E-tongue-MLR-SA model, selected from the 40 electrochemical signals recorded by E-tongue during analysis of each olive oil hydroethanolic extract.

c LOO-CV: leave-one-out cross validation procedure.

d Repeated $K$-fold-CV: cross-validation procedure with 4 folds, ensuring that at least $25 \%$ of the original data are used for internal validation, and 10 repetitions.

e CIELAB colour scale: $L^{*}$ value corresponds to lightness (0-black to 100perfect reflector diffuser); $\mathrm{a}^{*}$ negative value (green) and positive value (red); $b *$ negative values (blue) to positive values (yellow).

${ }^{\mathrm{f}}$ Sensory analysis was performed by a trained sensory panel comprising 8 panellists of the School of Agriculture of the Polytechnic Institute of Bragança (Portugal), following the IOC regulations [4,5] (intensity scale: from 0 (absence of attribute) to 10 (maximum attribute intensity)).

$\mathrm{g}$ E-tongue sensor signals included in the E-tongue-MLR-SA model: S1:3, S1:4, S1:6 to S1:8, S1:12, S1:13, S2:8, S2:10, S2:15, S2:16 and S2:20.

$\mathrm{h}$ E-tongue sensor signals included in the E-tongue-MLR-SA model: S1:4, S1:7, S1:10, S1:11, S1:13 to S1:15, S1:20, S2:2, S2:5, S2:7, S2:8 and S2:16.

${ }^{\mathrm{i}}$ E-tongue sensor signals included in the E-tongue-MLR-SA model: S1:4,
S1:12 to S1:14, S2:2, S2:4, S2:5, S2:7, S2:8, S2:15, S2:16 and S2:20.

$\mathrm{j}$ E-tongue sensor signals included in the E-tongue-MLR-SA model: S1:5, S1:8, S1:10, S1:13, S1:14, S1:19, S2:3, S2:8 to S2:10, S2:14, S2:16 and S2:20.

$\mathrm{k}$ E-tongue sensor signals included in the E-tongue-MLR-SA model: S1:6 to S1:9, S1:11, S1:12, S1:20, S2:1, S2:7, S2:8, S2:12, S2:18 and S2:19.

${ }^{1}$ E-tongue sensor signals included in the E-tongue-MLR-SA model: S1:5, S1:9, S1:11 to S1:13, S1:17, S2:2, S2:3, S2:5, S2:8, S2:11, S2:14 and S2:18.

$\mathrm{m}$ E-tongue sensor signals included in the E-tongue-MLR-SA model: S1:3, S1:5, S1:6, S1:11, S1:12, S1:15, S1:16, S1:19, S2:4, S2:6, S2:9, S2:11 and S2:12.

${ }^{\mathrm{n}}$ E-tongue sensor signals included in the E-tongue-MLR-SA model: S1:2, S1:6, S1:7, S1:11, S1:14, S1:15, S1:17, S1:20, S2:11, S2:15, S2:17, S2:18 and $\mathrm{S} 2: 20$.

${ }^{\circ}$ E-tongue sensor signals included in the E-tongue-MLR-SA model: S1:1 to S1:4, S1:7, S1:13, S1:18, S1:19, S2:5, S2:10, S2:11, S2:17, S2:19 and S2:20.

$\mathrm{P}$ E-tongue sensor signals included in the E-tongue-MLR-SA model: S1:1, S1:5, S1:6, S1:9, S1:10, S1:18, S1:20, S2:1, S2:5, S2:8, S2:14, S2:16 and S2:18.

q E-tongue sensor signals included in the E-tongue-MLR-SA model: S1:4 to S1:6, S1:12, S1:18, S2:2, S2:4, S2:5, S2:10, S2:13, S2:16, S2:17 and S2:20.

${ }^{r}$ E-tongue sensor signals included in the E-tongue-MLR-SA model: S1:1, S1:3, S1:5, S1:12, S1:13, S2:1 to S2:3, S2:5, S2:7, S2:8, S2:11, S2:18 and S2:20.

$s$ E-tongue sensor signals included in the E-tongue-MLR-SA model: S1:3 to $\mathrm{S} 1: 6, \mathrm{~S} 1: 11, \mathrm{~S} 1: 18$ to S1:20, S2:1, S2:3, S2:18 and S2:20.

t E-tongue sensor signals included in the E-tongue-MLR-SA model: S1:4, S1:5, $\mathrm{S} 1: 8, \mathrm{~S} 1: 13, \mathrm{~S} 1: 19, \mathrm{~S} 2: 3, \mathrm{~S} 2: 8$ to S2:11, S2:16, S2:17 and S2:20.

u E-tongue sensor signals included in the E-tongue-MLR-SA model: S1:4, S1:8, S1:10, S1:12, S1:20, S2:1, S2:3, S2:4, S2:11, S2:14 and S2:18.

$\mathrm{v}$ E-tongue sensor signals included in the E-tongue-MLR-SA model: S1:3, S1:5, S1:7, S1:10, S1:11, S1:16, S2:8, S2:10, S2:14, S2:15 and S2:19.

${ }^{\mathrm{w}}$ E-tongue sensor signals included in the E-tongue-MLR-SA model: S1:5, $\mathrm{S} 1: 8, \mathrm{~S} 1: 10, \mathrm{~S} 1: 11, \mathrm{~S} 1: 13$ to S1:15, S1:18, S2:2, S2:7, S2:9, S2:14 and S2:18.

${ }^{\mathrm{x}}$ E-tongue sensor signals included in the E-tongue-MLR-SA model: S1:2, S1:3, S1:10, S1:11, S1:13, S1:17, S1:18, S1:20, S2:3, S2:10, S2:12 and S2:14.

tocopherols contents, colour parameters as well as the intensity of positive gustatory-retronasal sensory sensations is reported and further compared with the few works available in the literature, regarding this field.

3.2.1. Peroxide value, oxidative stability, total phenols and total tocopherols contents

The PV is the most commonly used parameter for assessing primary oxidation products (i.e., the amount of hydroperoxides) in olive oil. Although, alone, this parameter should not be used to establish oil quality, since the hydroperoxides naturally decompose during storage, it is a suitable parameter to measure quality decrease over time. The possibility of assessing the PV level based on a potentiometric profile, recorded by the E-tongue, together with MLRM coupled with SA algorithm, was evaluated, using the PV data of olive oils produced from olive trees from different olive cultivars obtained by titration according to official methods. The results clearly showed (Table 2) that a MLRMSA-E-tongue model, based on the signals recorded by 12 sensors, which were selected using the SA algorithm, could be established and allowed satisfactorily predicting the PV of olive oils (repeated K-fold-CV: $\mathrm{R}^{2}$ $=0.95 \pm 0.05$; RMSE $=0.27 \pm 0.10 \mathrm{mEq} \mathrm{O}_{2} / \mathrm{kg}$ of olive oil). Furthermore, a deeper statistical analysis (Table 3 and Fig. 3) enabled concluding that the proposed MLRM-SA-E-tongue model allowed quantifying the PV with similar analytical accuracy as that achieved with the official titration technique (the slope and intercept values of the LR of the E-tongue predicted data versus the titration experimental values could be considered equal to one and zero, from a statistical point of view $[43,44])$. This satisfactory performance is in accordance with the results reported by Rodrigues et al. [20] and Semenov et al. [28], which also successfully used potentiometric E-tongues for assessing the PV of EVOO during storage or of vegetable oils (including olive oils), respectively.

The overall olive oil quality from production to consumption, and so its shelf-life, is highly dependent on the oil oxidative stability (OS) and the impact on the evolution of flavour, taste, colour, and the content of 
Table 3

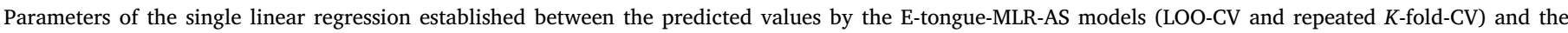

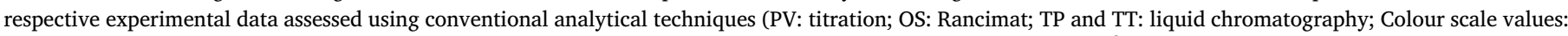

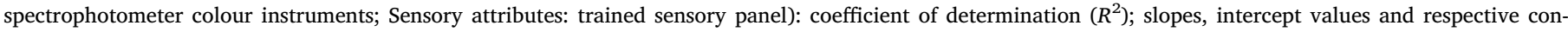
fidence intervals (CI) at 95\%.

\begin{tabular}{|c|c|c|c|c|c|c|c|c|c|c|}
\hline \multirow[t]{2}{*}{ Phenolic compounds } & \multicolumn{5}{|c|}{ LOO-CV } & \multicolumn{5}{|c|}{ Repeated K-fold-CV ${ }^{b}$} \\
\hline & $R^{2}$ & Slope & Slope $\mathrm{CI}^{\mathrm{c}}$ & Intercept & Intercept $\mathrm{CI}^{\mathrm{d}}$ & $R^{2}$ & Slope & Slope $\mathrm{CI}^{\mathrm{c}}$ & Intercept & Intercept $\mathrm{CI}^{\mathrm{d}}$ \\
\hline \multicolumn{11}{|l|}{ Physicochemical parameters } \\
\hline Peroxide value (mEq $\mathrm{O}_{2} / \mathrm{kg}$ olive oil) & 0.97 & 0.98 & {$[0.90,1.06]$} & 0.04 & {$[-0.23,0.31]$} & 0.94 & 1.00 & {$[0.96,1.03]$} & -0.01 & {$[-0.13,0.10]$} \\
\hline Oxidative stability (h) & 0.98 & 1.00 & {$[0.91,1.08]$} & 0.03 & {$[-2.0,2.1]$} & 0.91 & 0.97 & {$[0.93,1.01]$} & 0.63 & {$[-0.29,1.56]$} \\
\hline Total phenols (mg CAE/kg olive oil) & 0.98 & 0.97 & {$[0.87,1.06]$} & 13 & {$[-20,46]$} & 0.88 & 0.98 & {$[0.93,1.02]$} & 8.4 & {$[-7.8,24]$} \\
\hline Total tocopherols (mg/kg olive oil) & 0.98 & 1.02 & {$[0.98,1.05]$} & -5.4 & {$[-15,4.1]$} & 0.90 & 1.03 & {$[0.98,1.07]$} & -7.8 & {$[-20,4.5]$} \\
\hline \multicolumn{11}{|l|}{ Colour scale } \\
\hline \multirow[t]{3}{*}{ CIELAB scale $^{e}$} & 0.99 & 1.01 & {$[0.93,1.08]$} & -0.45 & {$[-5.2,4.3]$} & 0.96 & 1.00 & {$[0.98,1.03]$} & -0.19 & {$[-1.9,1.5]$} \\
\hline & 0.97 & 0.97 & {$[0.86,1.08]$} & -0.43 & {$[-2.0,1.1]$} & 0.89 & 0.99 & {$[0.94,1.03]$} & -0.20 & {$[-0.84,0.44]$} \\
\hline & 0.98 & 0.98 & {$[0.90,1.06]$} & 1.4 & {$[-4.1,6.9]$} & 0.92 & 0.99 & {$[0.95,1.03]$} & 0.47 & {$[2.2,3.1]$} \\
\hline \multicolumn{11}{|c|}{ Sensory gustatory-retronasal positive sensations ${ }^{\mathrm{f}}$} \\
\hline Green intensity sensation & 0.99 & 1.01 & {$[0.95,1.07]$} & -0.09 & {$[-0.54,0.37]$} & 0.97 & 1.01 & {$[0.98,1.03]$} & -0.05 & {$[-0.22,0.12]$} \\
\hline Sweet intensity sensation & 0.97 & 0.90 & {$[0.80,0.99]$} & 0.14 & {$[-0.11,0.39]$} & 0.84 & 0.84 & {$[0.80,0.89]$} & 0.32 & {$[0.00,0.19]$} \\
\hline Bitter intensity sensation & 0.98 & 1.00 & {$[0.92,1.08]$} & 0.04 & {$[-0.40,0.47]$} & 0.91 & 1.00 & {$[0.96,1.05]$} & 0.01 & {$[-0.22,0.24]$} \\
\hline Pungent intensity sensation & 0.99 & 1.00 & {$[0.93,1.06]$} & 0.01 & {$[-0.34,0.37]$} & 0.92 & 0.96 & {$[0.92,1.00]$} & 0.22 & {$[0.00,0.43]$} \\
\hline Tomato intensity sensation & 0.98 & 0.98 & {$[0.90,1.07]$} & 0.10 & {$[-0.38,0.58]$} & 0.90 & 1.01 & {$[0.97,1.06]$} & -0.02 & {$[-0.26,0.22]$} \\
\hline Apple intensity sensation & 0.99 & 1.00 & {$[0.97,1.02]$} & 0.01 & {$[-0.08,0.10]$} & 0.95 & 1.00 & {$[0.98,1.03]$} & 0.01 & {$[-0.08,0.11]$} \\
\hline Banana intensity sensation & 0.99 & 1.00 & {$[0.98,1.02]$} & 0.00 & {$[-0.05,0.06]$} & 0.96 & 0.94 & {$[0.91,0.96]$} & 0.20 & {$[0.13,0.27]$} \\
\hline Dry fruits intensity sensation & 0.97 & 1.02 & {$[0.91,1.14]$} & -0.05 & {$[-0.35,0.25]$} & 0.90 & 0.98 & {$[0.93,1.02]$} & 0.12 & {$[0.01,0.23]$} \\
\hline Fresh herbs intensity sensation & 0.94 & 1.00 & {$[0.83,1.16]$} & -0.05 & {$[-0.80,0.70]$} & 0.89 & 0.98 & {$[0.93,1.02]$} & 0.09 & {$[-0.12,0.30]$} \\
\hline Tomato leaves intensity sensation & 0.99 & 1.00 & {$[0.98,1.01]$} & 0.01 & {$[-0.06,0.07]$} & 0.96 & 1.00 & {$[0.98,1.03]$} & -0.04 & {$[-0.15,0.06]$} \\
\hline Cabbage intensity sensation & 0.97 & 1.01 & {$[0.90,1.1]$} & 0.06 & {$[-0.53,0.65]$} & 0.89 & 1.03 & {$[0.98,1.08]$} & 0.02 & {$[-0.22,0.26]$} \\
\hline
\end{tabular}

a LOO-CV (leave-one-out cross-validation).

b Repeated $K$-fold-CV (4 folds $\times 10$ repeats).

c $95 \%$ slope confidence interval.

d $95 \%$ intercept confidence interval.

e CIELAB colour scale: $L^{*}$ value corresponds to lightness (0-black to 100 -perfect reflector diffuser); $a *$ negative value (green) and positive value (red); $b *$ negative values (blue) to positive values (yellow).

${ }^{\mathrm{f}}$ Sensory analysis was performed by a trained sensory panel comprising 8 panellists of the School of Agriculture of the Polytechnic Institute of Bragança (Portugal), following the IOC regulations [4,5] (intensity scale: from 0 (absence of attribute) to 10 (maximum attribute intensity)).

endogenous antioxidants and other minor constituents beneficial to health. The olive oil oxidative stability may be determined using the Rancimat method [20], which may be a time-consuming task. Previously, Rodrigues et al. [20] demonstrated the capability of a similar potentiometric E-tongue for assessing the OS of EVOO during one year of storage. Thus, in this work it was intended to verify the possibility of using a potentiometric-chemometric approach to determine the OS of olive oils from diverse olive cultivars. The results (Tables 2-3, Fig. 3) pointed out that a MRLM-SA-E-tongue model, which used the potentiometric signals recorded by 13 sensors (selected by the SA algorithm), could be used to predict the OS of the studied olive oils (repeated K-fold-CV: $\mathrm{R}^{2}=0.95 \pm 0.05$; RMSE $=1.7 \pm 0.8 \mathrm{~h}$ ) and with an accuracy similar, from a statistical point of view, to that of the Rancimat method [43,44]. This E-tongue performance is in agreement with the previously mentioned report from Rodrigues et al. [20].

The total phenols (TP) contents, which are usually assessed using the Folin- Ciocalteau spectrophotometric method, were determined in this work by calculating the sum of the individual bioactive phenolic compounds (i.e., the polar phenols) quantified by HPLC on the olive oils studied. This evaluation is of major relevance since polar phenols are related with beneficial health and sensorial properties, while also contributing for olive oil resistance to oxidation. Previous works have reported the successful use of potentiometric E-tongues for assessing the TP levels in Arbequina olive oils [18] and on vegetable oils [28]. So, the capability of the novel potentiometric E-tongue device was also studied for this specific application. Once again, the results (Tables 2, 3, Fig. 3) confirmed the possibility of using a MRLM-SA-E-tongue model, based on signal profiles of 12 sensors, for predicting the TP contents of olive oils from different olive cultivars (repeated K-fold-CV: $\mathrm{R}^{2}$ $=0.92 \pm 0.07 ; \mathrm{RMSE}=22 \pm 8 \mathrm{mg} \mathrm{CAE} / \mathrm{kg}$ of olive oil). The quality of the results indicates that the E-tongue-based procedure had, from a statistical point of view, a similar accuracy compared to the HPLC conventional method, confirming the reported successful performance of these type of potentiometric devices for TP evaluation [18,28]. Furthermore, the potentiometric E-tongue predictive performance was comparable to that achieved with voltammetric E-tongues, described in the literature $[11,14,15]$.

Tocopherols are non-polar phenols, which also contribute to several of the beneficial nutritional and health effects related to the olive oil consumption and olive oil stability, due to their antioxidant activity. Olive oil extraction using hydroethanolic solutions (water: ethanol, $80: 20, \mathrm{v} / \mathrm{v}$ ) would promote the extraction of polar compounds from the oil, Semenov et al. [28], showed that it was possible to satisfactorily determine the total tocopherols (TT) contents of vegetable oils by analysing their water-isopropyl alcohol extracts. Indeed, the presence of small alcohol amounts increases tocopherols solubility due to nonpolar attractive interactions with the alcohol, being the tocopherol concentrations in the hydroalcoholic phase in a relative equilibrium to that of the olive oil [40], and therefore potentially enabling quantification. Thus, in the present work, the potentiometric E-tongue with a novel cylindrical shape, comprising lipid membranes with cross-sensitivity, was applied for the first time for evaluating the TT contents of olive oils produced from olive trees of different cultivars. The results (Tables 2, 3; Fig. 3) proved that a MRLM-SA-E-tongue model could be established for predicting the TT contents (repeated K-fold-CV: $\mathrm{R}^{2}=0.93 \pm 0.07$; RMSE $=28 \pm 16 \mathrm{mg} / \mathrm{kg}$ of olive oil) with a similar accuracy to that 

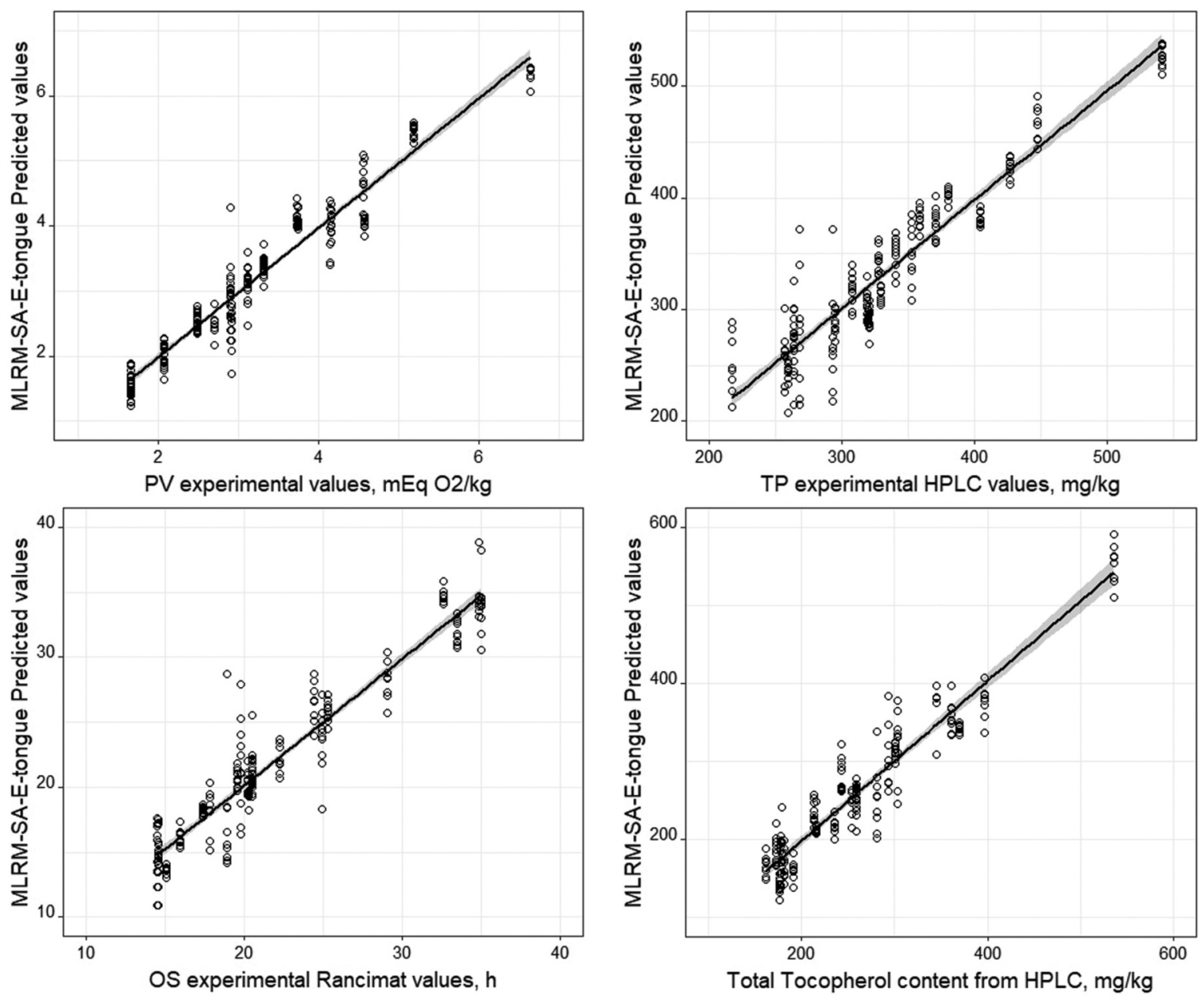

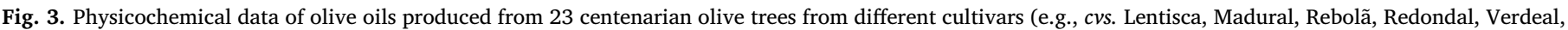

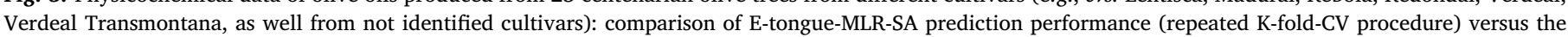
experimental values (peroxide values by titration; oxidative stability according to the Rancimat assays; total phenols and total tocopherols by HPLC).

achieved with the HPLC technique, based on the statistical evaluation of the regression parameters $[43,44]$, as well as with that reported by Semenov et al. [28], for quantifying TT levels in vegetable oils using a potentiometric E-tongue or by Vasilescu et al. [32], in the determination of the individual tocopherol contents, using a voltammetric device.

Finally, the results shown in Tables 2 and 3, also pointed out the more overoptimistic results achieved when the LOO-CV variant was used to check the predictive performance (i.e., the internal cross-validation results). This observation shows the need of applying more robust $\mathrm{CV}$ variants, such as the repeated $\mathrm{K}-$ fold-CV, for verifying the possibility of using a potentiometric E-tongue as a practical, fast and single-assay technique as a complementary or even alternative approach to the standard conventional analytical techniques usually applied for olive oil physicochemical evaluation (i.e., titration, Rancimat or chromatographic methods).

\subsubsection{Colour scale CIELAB values}

Olive oil colour is one intrinsic attribute that may affect the consumers' choice when buying this type of high-value food [49,50], although several other extrinsic attributes, like geographical origin and designation, organic certification and price, may also strongly affect the consumers' preferences. Olive oil colour is mostly the combined result of chlorophylls presence and its degradation products, the pheophytins, together with carotenoids [41]. The assessment of the olive oil colour plays a key role on the olive oil commercialization from the consumer's perspective, although not regarded as an official quality parameter. The CIELAB colour scale may be used for characterizing the olive oil colour through the determination of the $L^{*}, a^{*}$ and $b^{*}$ values, which are related to the lightness, green-red and blue-yellow parameters, respectively. The values are experimentally determined using colorimeters, requiring this additional equipment, if an overall characterization of an olive oil sample is intended. Chlorophylls and carotenoids quantification in olive oil organic extracts, using a voltammetric E-tongue, has been previously reported [30]. Therefore, the possibility of assessing the $L^{*}, a^{*}$ and $b^{*}$ values of the CIELAB scale using the novel potentiometric E-tongue was evaluated for the first time. As can be inferred from Table 2 as well as from Fig. 4, it was possible to establish MRLM-SA-E-tongue models, based on the potentiometric data gathered from different sub-sets of 13 sensors (selected by the SA algorithm) that could be used to satisfactorily predict the three parameters of the CIELAB colour scale (repeated K-fold-CV: $0.93 \pm 0.06 \leq \mathrm{R}^{2} \leq$ $0.97 \pm 0.03)$. The monochromatic variables were determined using a Konica Minolta colorimeter (model CR-400), following the method described by Zamora et al. [51]. This capability could be tentatively attributed to the interactions established between the lipid membranes (E-tongue) and the olive oil pigments extracted with the hydroethanolic solution, namely chlorophylls and carotenoids, which are responsible for the greenness and yellowness oil colours [41]. Also, from Table 3 it 

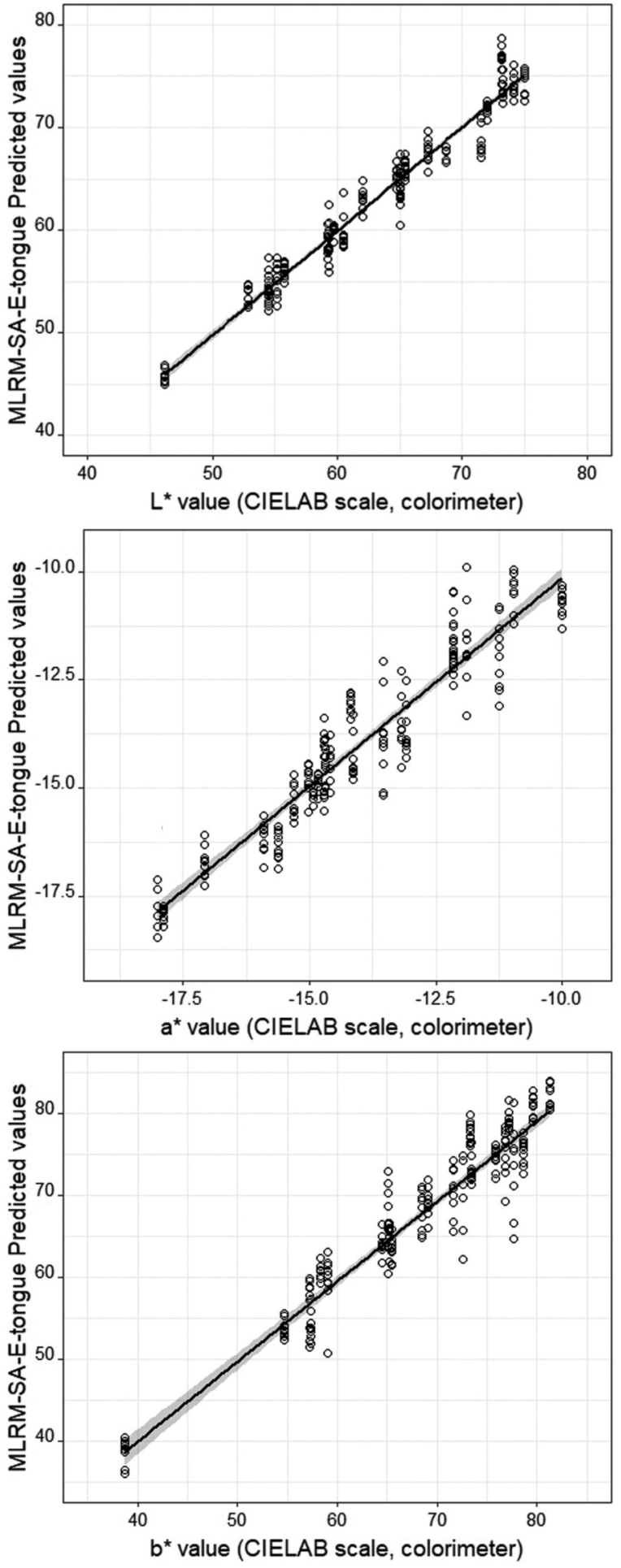

Fig. 4. CIELAB colour scale data ( $L^{*}$, $a^{*}$ and $b^{*}$ values) of olive oils produced from 23 centenarian olive trees from different cultivars (e.g., cvs. Lentisca, Madural, Rebolã, Redondal, Verdeal, Verdeal Transmontana, as well from not identified cultivars): comparison of E-tongue-MLR-SA prediction performance (repeated K-fold-CV procedure) versus the experimental values (colorimeter assays).

is clear that the E-tongue together with chemometric tools is an analytical procedure with a similar accuracy as that achieved with a colorimeter, based on the statistical analysis regarding the slope and intercept values of the LR established between predicted versus experimental data $[43,44]$.

Once again, the data also pointed out the overoptimistic results achieved when applying the LOO-CV as well as the need of using a more robust $\mathrm{CV}$ variant for this kind of analysis.

\subsubsection{Sensory gustatory-retronasal positive attributes}

The sensory attributes of olive oils may also greatly influence not only the olive oil quality grade but also the consumers' preference. Indeed, besides the physicochemical levels that must be fulfilled for an olive oil to be classified as EVOO or VOO, the fruitiness intensity must be greater than 0 and no defects should be perceived or the maximum intensity of the perceived defects should be lower than 3.5 in a $0-10$ intensity scale, respectively [2-5]. On the other hand, the perception of other positive attributes (e.g, green, bitter, or herbs/fruits/dry fruits sensations) and their intensities may have an important commercial value, allowing supplying differentiated organoleptic profiles, fulfilling the new trend demands of consumers. However, olive oil sensory evaluation requires an official sensory panel, which poses several limitations, like, panel availability, panellists score subjectivity, analysis cost and the low number of samples that can be daily evaluated. So, in the last years several voltammetric and potentiometric devices have been proposed for the organoleptic evaluation of olive oils or table olives, including both positive $[22,23,39,52-54]$ and negative attributes $[50,55,56]$. Based on the reported satisfactory performances of E-tongues as taste sensor devices, in this work, the application of the novel potentiometric E-tongue to assess the intensity of positive gustatoryretronasal sensations was evaluated and further checked by comparing with the results of a trained sensory panel. The results (Table 2 and Fig. 5) allowed verifying the capability of establishing MLRM-SA-Etongue models (based on different sub-sets of 11-14 sensors) for predicting the intensities of 11 positive attributes of olive oils from different cultivars, some of them assessed for the first time using an electrochemical approach (repeated K-fold-CV: $0.90 \pm 0.07 \leq \mathrm{R}^{2} \leq$ $0.98 \pm 0.02 ; 0.15 \pm 0.06 \leq$ RMSE $\leq 0.64 \pm 0.32$ ). It should be noticed that, besides the common green, sweet, bitter, and pungent sensations, the E-tongue could be used to evaluate quite distinct positive sensations such as tomato and tomato leaves, apple, banana, cabbage, dry fruits and fresh herbs, showing its high versatility as a taste sensor device. Furthermore, based on the evaluation of the parameters (slope and intercept values) of the LR of predicted versus experimental data (Table 3) it can be stated that, in general, the potentiometric Etongue delivered a representative descriptive fingerprint of the positive sensory attributes of the olive oils evaluated with a similar accuracy of that achieved by a trained sensory panel.

Once more, the results also pointed out that the LOO-CV procedure is overoptimistic, showing the need of implementing more robust $\mathrm{CV}$ procedures, like the repeated $\mathrm{K}$-fold-CV variant, for assessing the predictive quantitative performance of E-tongue based models.

\section{Conclusions}

The present study confirmed and in some cases demonstrated for the first time that, a potentiometric E-tongue, comprising non-specific and cross-sensitive lipid polymeric sensor membranes, together with chemometric tools, could be used as a practical analytical approach for the simultaneous assessment, in a single-assay, of several physicochemical and sensory data, on a single-assay basis of olive oil hydroethanolic extracts. Indeed, the device was able to predict, based on a single-run potentiometric assay, characteristics so diverse as peroxide value, oxidative stability, total phenols and tocopherols contents, CIELAB colour values and the intensity of 11 positive gustatory-retronasal sensations, which unequivocally demonstrates the versatility and utility of such a device. The overall satisfactory results reported in this work, as well as, the satisfactory performances reported in the literature regarding the use of potentiometric E-tongue comprising lipid polymeric sensor membranes, for both qualitative and quantitative analysis of olive oils, will contribute, hopefully, to turn real the use of this sensor-based strategy for routine olive oils evaluation at industrial level. 

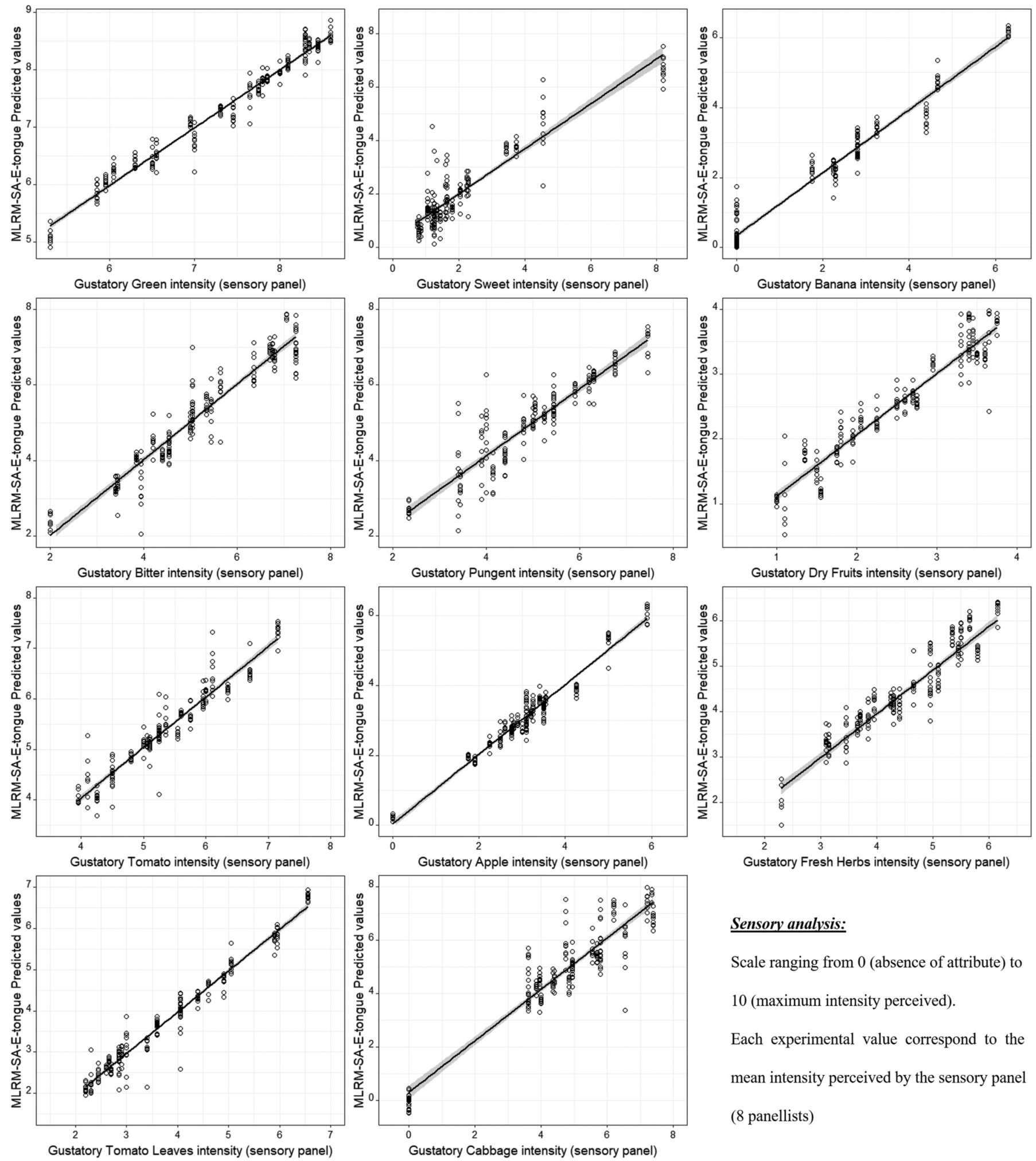

\section{Sensory analysis:}

Scale ranging from 0 (absence of attribute) to

10 (maximum intensity perceived).

Each experimental value correspond to the mean intensity perceived by the sensory panel

(8 panellists)

Fig. 5. Gustatory-retronasal positive attributes intensities (green, sweet, bitter, pungent, tomato, apple, banana, dry fruits, fresh herbs, tomato leaves, cabbage sensations and harmony overall perception) of olive oils produced from 23 centenarian olive trees from different cultivars (e.g., cvs. Lentisca, Madural, Rebolã, Redondal, Verdeal, Verdeal Transmonstana, as well from not identified cultivars): comparison of E-tongue-MLR-SA prediction performance (repeated K-fold-CV procedure: 4 folds $\times 10$ repeats; each fold corresponding to the data of 5- 6 independent olive trees) versus the sensations intensities perceived by the trained panellists. 


\section{Acknowledgments}

This work was financially supported by Project POCI-01-0145FEDER-006984 - Associate Laboratory LSRE-LCM, Project UID/BIO/ 04469/2013 - CEB, Project UID/QUI/50006/2013 - REQUIMTE-LAQV and strategic project PEst-OE/AGR/UI0690/2014 - CIMO all funded by European Regional Development Fund (ERDF) through COMPETE2020 - Programa Operacional Competitividade e Internacionalização (POCI) - and by national funds through FCT - Fundação para a Ciência e a Tecnologia I.P. Ítala G. Marx also acknowledges the research grant provided by Project UID/EQU/50020/2013 and POCI-01-0145-FEDER006984.

\section{Conflict of interest}

The authors declare that they have no conflict of interest.

\section{Ethical approval}

This article does not contain any studies with human participants or animals performed by any of the authors.

\section{Informed consent}

Not applicable.

\section{References}

1] G. Buckland, C.A. Gonzalez, The role of olive oil in disease prevention: a focus on the recent epidemiological evidence from cohort studies and dietary intervention trials, Br. J. Nutr. 113 (2015) S94-S101, https://doi.org/10.1017/ S0007114514003936.

[2] EU No 1348/2013, Commission implementing regulation of 16 December 2013 amending Regulation No 2568/91/EEC on the characteristics of olive oil and oliveresidue oil and on the relevant methods of analysis, Off. J. Eur. Union L338 (2013) $31-67$.

[3] EU No 61/2011, Commission regulation of 24 January 2011 amending regulation No 2568/91/EEC on the characteristics of olive oil and olive pomace oil and on the relevant methods of analysis, Off. J. Eur. Union L23 (2011) 1-13.

[4] IOC, International Olive Council, Sensory analysis of olive oil - Method for the organoleptic assessment of virgin olive oil, COI/T.20/Doc. No 15/Rev. 6 November 2013, p. 18. (〈http://www.internationaloliveoil.org/〉).

[5] IOC, International Olive Council, IOC Mario Solinas quality award - Rules of the International competition for extra virgin olive oils. T.30/Doc. No 17 June 2014, p. 9. 〈http://www.internationaloliveoil.org/ $>$.

[6] A. Mastralexi, N. Nenadis, M. Tsimidou, Addressing analytical requirements to support health claims on "olive oil polyphenols" (EC Regulation 432/2012), J. Agric. Food Chem. 62 (2014) 2459-2461, https://doi.org/10.1021/jf5005918.

[7] R. Aparicio, M.T. Morales, R. Aparicio-Ruiz, N. Tena, D.L. García-González, Authenticity of olive oil: mapping and comparing official methods and promising alternatives, Food Res. Int. 54 (2013) 2025-2038, https://doi.org/10.1016/j. foodres.2013.07.039.

[8] Yu Vlasov, A. Legin, A. Rudnitskaya, C. Di Natale, A. D’Amico, Nonspecific sensor arrays ("electronic tongue") for chemical analysis of liquids: (IUPAC technical report), Pure Appl. Chem. 77 (2005) 1965-1983, https://doi.org/10.1351/ pac200577111965.

[9] T. Majchrzak, W. Wojnowski, T. Dymerski, J. Gębicki, J. Namieśnik, Electronic noses in classification and quality control of edible oils: a review, Food Chem. 246 (2018) 192-201, https://doi.org/10.1016/j.foodchem.2017.11.013.

[10] C. Apetrei, Novel method based on polypyrrole-modified sensors and emulsions for the evaluation of bitterness in extra virgin olive oils, Food Res. Int. 48 (2012) 673-680, https://doi.org/10.1016/j.foodres.2012.06.010.

[11] I.M. Apetrei, C. Apetrei, Voltammetric e-tongue for the quantification of total polyphenol content in olive oils, Food Res. Int. 54 (2013) 2075-2082, https://doi. org/10.1016/j.foodres.2013.04.032.

[12] I.M. Apetrei, C. Apetrei, Detection of virgin olive oil adulteration using a voltammetric e-tongue, Comput. Electron. Agric. 108 (2014) 148-154, https://doi.org/10. 1016/j.compag.2014.08.002

[13] C. Apetrei, M.L. Rodríguez-Méndez, J.A. de Saja, Modified carbon paste electrodes for discrimination of vegetable oils, Sens. Actuators B Chem. 111-112 (2005) 403-409, https://doi.org/10.1016/j.snb.2005.03.041.

[14] C. Apetrei, F. Gutierez, M.L. Rodríguez-Méndez, J.A. de Saja, Novel method based on carbon paste electrodes for the evaluation of bitterness in extra virgin olive oils, Sens. Actuators B Chem. 121 (2007) 567-575, https://doi.org/10.1016/j.snb.2006. 04.091.

[15] M.L. Rodríguez-Méndez, C. Apetrei, J.A. de Saja, Evaluation of the polyphenolic content of extra virgin olive oils using an array of voltammetric sensors,
Electrochim. Acta 53 (2008) 5867-5872, https://doi.org/10.1016/j.electacta.2008. 04.006.

[16] P. Oliveri, M.A. Baldo, S. Daniele, M. Forina, Development of a voltammetric electronic tongue for discrimination of edible oils, Anal. Bioanal. Chem. 395 (2009) 1135-1143, https://doi.org/10.1007/s00216-009-3070-8.

[17] Z. Haddi, H. Alami, N. El Bari, M. Tounsi, H. Barhoumi, A. Maaref, N. JaffrezicRenault, B. Bouchikhi, Electronic nose and tongue combination for improved classification of Moroccan virgin olive oil profiles, Food Res. Int. 54 (2013) 1488-1498, https://doi.org/10.1016/j.foodres.2013.09.036.

[18] T.H. Borges, A.M. Peres, L.G. Dias, I. Seiquer, J.A. Pereira, Application of a potentiometric electronic tongue for assessing phenolic and volatile profiles of Arbequina extra virgin olive oils, LWT - Food Sci. Technol. 93 (2018) 150-157, https://doi.org/10.1016/j.lwt.2018.03.025.

[19] L.G. Dias, A. Fernandes, A.C.A. Veloso, A.A.S.C. Machado, J.A. Pereira, A.M. Peres, Single-cultivar extra virgin olive oil classification using a potentiometric electronic tongue, Food Chem. 160 (2014) 321-329, https://doi.org/10.1016/j.foodchem. 2014.03.072.

[20] N. Rodrigues, L.G. Dias, A.C.A. Veloso, J.A. Pereira, A.M. Peres, Monitoring olive oils quality and oxidative resistance during storage using an electronic tongue, LWT - Food Sci. Technol. 73 (2016) 683-692, https://doi.org/10.1016/j.lwt.2016.07. 002.

[21] F. Souayah, N. Rodrigues, A.C.A. Veloso, L.G. Dias, J.A. Pereira, S. Oueslati, A.M. Peres, Discrimination of olive oil by cultivar, geographical origin and quality using potentiometric electronic tongue fingerprints, J. Am. Oil Chem. Soc. 94 (2017) 1417-1429, https://doi.org/10.1007/s11746-017-3051-6.

[22] A.C.A. Veloso, L.G. Dias, N. Rodrigues, J.A. Pereira, A.M. Peres, Sensory intensity assessment of olive oils using an electronic tongue, Talanta 146 (2016) 585-593, https://doi.org/10.1016/j.talanta.2015.08.071.

[23] S. Slim, N. Rodrigues, L.G. Dias, A.C.A. Veloso, J.A. Pereira, S. Oueslati, A.M. Peres, Application of an electronic tongue for Tunisian olive oils' classification according to olive cultivar or physicochemical parameters, Eur. Food Res. Technol. 243 (2017) 1459-1470, https://doi.org/10.1007/s00217-017-2856-8.

[24] U. Harzalli, N. Rodrigues, A.C.A. Veloso, L.G. Dias, J.A. Pereira, S. Oueslati, A.M. Peres, A taste sensor device for unmasking admixing of rancid or winey-vinegary olive oil to extra virgin olive oil, Comput. Elelctron. Agr. 144 (2018) 222-231, https://doi.org/10.1016/j.compag.2017.12.016.

[25] R. Prata, J.A. Pereira, N. Rodrigues, L.G. Dias, A.C.A. Veloso, S. Casal, A.M. Peres, Olive oil total phenolic contents and sensory sensations trends during oven and microwave heating processes and their discrimination using an electronic tongue (Article ID 7826428), J. Food Qual. 2018 (2018), https://doi.org/10.1155/2018/ 7826428.

[26] N. Rodrigues, L. Oliveira, L. Mendanha, M. Sebti, L.G. Dias, S. Oueslati, A.C.A. Veloso, J.A. Pereira, A.M. Peres, Olive oil quality and sensory changes during house-use simulation and temporal assessment using an electronic tongue, J. Am. Oil Chem. Soc. 95 (2018) 1121-1137, https://doi.org/10.1002/aocs.12093.

[27] T.A. Enache, A. Amine, C.M.A. Brett, A.M. Oliveira-Brett, Virgin olive oil orthophenols - electroanalytical quantification, Talanta 105 (2013) 179-186, https://doi org /10.1016/j.talanta.2012.11.055.

[28] V. Semenov, S. Volkov, M. Khaydukova, A. Feforav, I. Lisitsyn, D. Kirsanov, A. Legin, Determination of three quality parameters in vegetable oils using potentiometric e-tongue, J. Food Compos. Anal. 75 (2019) 75-80, https://doi.org/10. 1016/j.jfca.2018.09.015.

[29] E. Fernández, L. Vidal, A. Canals, Rapid determination of hydrophilic phenols in olive oil by vortex-assisted reversed-phase dispersive liquid-liquid microextraction and screen-printed carbon electrodes, Talanta 181 (2018) 44-51, https://doi.org/ 10.1016/j.talanta.2017.12.075.

[30] K. Tahri, A.A. Duarte, G. Carvalho, P.A. Ribeiro, M.G. Silva, D. Mendes, Distinguishment, identification and aroma compound quantification of Portuguese olive oils based on physicochemical attributes, HS-GC/MS analysis and voltammetric electronic tongue, J. Sci. Food Agric. 98 (2018) 681-690, https://doi.org/ $10.1002 /$ jsfa. 8515 .

[31] T.G. Diaz, A. Espinosa-Mansilla, B.R. Murillo, F. Salinas, Voltammetric behavior and determination of nordihydroguaiaretic acid in presence of other antioxidants using PLS calibration, Electroanalysis 15 (2003) 646-651, https://doi.org/10.1002/elan. 200390081.

[32] I. Vasilescu, S.A.V. Eremia, C. Albu, A. Radoi, S.-C. Litescu, G.-L. Radu, Determination of the antiradical properties of olive oils using an electrochemical method based on DPPH radical, Food Chem. 166 (2015) 324-329, https://doi.org/ 10.1016/j.foodchem.2014.06.042.

[33] N. Rodrigues, S. Casal, T. Pinho, A.M. Peres, A. Bento, P. Baptista, J.A. Pereira, Ancient olive trees as a source of olive oils rich in phenolic compounds, Food Chem. 276 (2019) 231-239.

[34] IOC, International Olive Council, Chemical analysis of olive oils, MethodDetermination of Biophenols in olive oil by HPLC, COI/T.20/Doc No 29/Rev.1, 2017.

[35] ISO 9936, Animal and Vegetable Fats and Oils - Determination of Tocopherol and Tocotrienol Contents by High-Performance Liquid Chromatography, 2006.

[36] N. Rodrigues, R. Malheiro, S. Casal, M.C. Asensio-S.-Manzanera, A. Bento, J.A. Pereira, Influence of spike lavender (Lavandula latifolia Med.) essential oil in the quality, stability and composition of soybean oil during microwave heating, Food Chem. Toxicol. 50 (2012) 2894-2901, https://doi.org/10.1016/j.fct.2012.05 020 .

[37] P. Limón, R. Malheiro, S. Casal, F.G. Acién-Fernández, J.M. Fernández-Sevilla, N. Rodrigues, R. Cruz, R. Bermejo, J.A. Pereira, Improvement of stability and carotenoids fraction of virgin olive oils by addition of microalgae Scenedesmus almeriensis extracts, Food Chem. 175 (2015) 203-211, https://doi.org/10.1016/j. 
foodchem.2014.10.150.

[38] Y. Kobayashi, M. Habara, H. Ikezazki, R. Chen, Y. Naito, K. Toko, Advanced taste sensors based on artificial lipids with global selectivity to basic taste qualities and high correlation to sensory scores, Sensors 10 (2010) 3411-3443, https://doi.org/ $10.3390 / \mathrm{s} 100403411$.

[39] C. Apetrei, I.M. Apetrei, S. Villanueva, J.A. de Saja, F. Gutierrez-Rosales, M.L. Rodriguez-Mendez, Combination of an e-nose, an e-tongue and an e-eye for the characterisation of olive oils with different degree of bitterness, Anal. Chim. Acta 663 (2010) 91-97, https://doi.org/10.1016/j.aca.2010.01.034.

[40] M.D. Dubbs, R.B. Gupta, Solubility of vitamin E ( $\alpha$-Tocopherol) and vitamin K3 (Menadione) in ethanol-water mixture, J. Chem. Eng. Data 43 (1998) 590-591, https://doi.org/10.1021/je980017l.

[41] M.J. Moyano, F.J. Heredia, A.J. Meléndez-Martínez, The color of olive oils: the pigments and their likely health benefits and visual and instrumental methods of analysis, Compr. Rev. Food Sci. Food Saf. 9 (2010) 278-291, https://doi.org/10. 1111/j.1541-4337.2010.00109.x.

[42] L.G. Dias, C. Sequeira, A.C.A. Veloso, M.E.B.C. Sousa, A.M. Peres, Evaluation of healthy and sensory indexes of sweetened beverages using an electronic tongue, Anal. Chim. Acta 848 (2014) 32-42, https://doi.org/10.1016/j.aca.2014.08.003.

[43] B. Roig, O. Thomas, UV monitoring of sugars during wine making, Carbohydr. Res. 338 (2003) 79-83, https://doi.org/10.1016/S0008-6215(02)00396-8.

[44] B. Roig, O. Thomas, Rapid estimation of global sugars by UV photodegradation and UV spectrophotometry, Anal. Chim. Acta 477 (2003) 325-329, https://doi.org/10. 1016/S0003-2670(02)01427-7.

[45] J. Cadima, J.O. Cerdeira, M. Minhoto, Computational aspects of algorithms for variable selection in the context of principal components, Comput. Stat. Data Anal. 47 (2004) 225-236, https://doi.org/10.1016/j.csda.2003.11.001.

[46] J. Cadima, J.O. Cerdeira, P.D. Silva, M. Minhoto, The subselect R package. 〈http:// cran.rproject.org/web/packages/subselect/vignettes/subselect.pdf/ $>$ (Accessed date: 15 February 2016)

[47] W.N. Venables, B.D. Ripley, Modern Applied Statistics With S (Statistics and Computing), 4th ed., Springer, New York, 2002.

[48] L.G. Dias, A.M. Peres, A.C.A. Veloso, F.S. Reis, M. Vilas Boas, A.A.S.C. Machado, An electronic tongue taste evaluation: identification goat milk adulterations with bovine milk, Sens. Actuators B Chem. 136 (2009) 209-217, https://doi.org/10. 1016/j.snb.2008.09.025

[49] I.A. van der Lans, K. van Ittersum, A. Cicco, M. Loseby, The role of the region of origin and EU certificates of origin in consumer evaluation of food products, Eur. Rev. Agric. Econ. 28 (2001) 451-477, https://doi.org/10.1093/erae/28.4.451.

[50] Y. Erraach, S. Sayadi, A.C. Gómez, C. Parra-López, Consumer-stated preferences towards protected designation of origin (PDO) labels in a traditional olive-oil-producing country: the case of Spain, New Medit. 13 (2014) 11-19.

[51] R. Zamora, C. Olmo, J.L. Navarro, F.J. Hidalgo, Contribution of phospholipid pyrrolization of the color reversion produced during deodorization of poorly degummed vegetable oils, J. Agric. Food Chem. 52 (2004) 4166-4171, https://doi. org/10.1021/jf049864k

[52] E. Borràs, J. Ferré, R. Boqué, M. Mestres, L. Aceña, A. Calvo, O. Busto, Prediction of olive oil sensory descriptors using instrumental data fusion and partial least squares (PLS) regression, Talanta 155 (2016) 116-123, https://doi.org/10.1016/j.talanta. 2016.04.040.

[53] M.L. Rodríguez-Méndez, C. Apetrei, J.A. de Saja, Electronic tongues purposely designed for the organoleptic characterization of olive oils, in: V.R. Preedy, R.R. Watson (Eds.), Olives and Olive Oil in Health and Disease Prevention, Academic Press, London, 2010, pp. 525-532, , https://doi.org/10.1016/B978-0-12 374420-3.00057-7.

[54] Í.M.G. Marx, N. Rodrigues, L.G. Dias, A.C.A. Veloso, J.A. Pereira, D.A. Drunkler, A.M. Peres, Quantification of table olives' acid, bitter and salty tastes using potentiometric electronic tongue fingerprints, LWT-Food Sci. Technol. 79 (2017) 394-401, https://doi.org/10.1016/j.lwt.2017.01.060.

[55] E. Borràs, J. Ferré, R. Boqué, M. Mestres, L. Aceña, A. Calvo, O. Busto, Olive oil sensory defects classification with data fusion of instrumental techniques and multivariate analysis (PLS-DA), Food Chem. 203 (2016) 314-322, https://doi.org/ 10.1016/j.foodchem.2016.02.038.

[56] Í.M.G. Marx N. Rodrigues, L.G. Dias, A.C.A. Veloso, J.A. Pereira, D.A. Drunkler, A.M. Peres, Assessment of table olives' organoleptic defects intensities based on the potentiometric fingerprint recorded by an electronic tongue, Food Bioprocess Technol. 10 (2017) 1310-1323, https://doi.org/10.1007/s11947-017-1902-7. 